\title{
Prevalência de dislipidemias e fatores de risco associados
}

\section{Prevalence of dyslipidemias and associated risk factors}

Lorena Braz de Oliveira ${ }^{1}$, Izabela Borges de Carvalho², Carla Solange de Melo Escórcio-Dourado ${ }^{3}$, José Charles Lima Dourado4, Matheus Oliveira do Nascimento ${ }^{1}$

1. Discente do curso de Farmácia da Universidade Federal do Piauí (UFPI), Teresina, PI, Brasil. 2. Mestranda do Programa de Pós-graduação em Farmacologia da Universidade Federal do Piauí (UFPI), Teresina, PI, Brasil. 3. Docente do curso de Farmácia da Universidade Federal do Piauí (UFPI), Teresina, PI, Brasil. 4. Docente do curso de Farmácia da Faculdade Santo Agostinho (FSA), Teresina, PI, Brasil.

\section{Resumo}

Introdução: A dislipidemia caracteriza-se pela elevação dos níveis plasmáticos de colesterol de baixa densidade (LDL-c), redução dos níveis de colesterol de alta densidade (HDL-c) e/ou aumento de triglicérides (TG). Essas alterações evidenciam o risco para doenças cardiovasculares (DCV) e uma alta probabilidade de ocorrência de morte por eventos coronarianos. Objetivo: Avaliar a prevalência de dislipidemia e fatores de risco associados em pacientes ambulatoriais do hospital universitário da Universidade Federal do Piauí. Métodos: Trata-se de um estudo transversal, no qual a amostra foi constituída por 124 pacientes. Foram analisados os principais fatores de risco modificáveis: sobrepeso e sedentarismo, e não modificáveis: idade e sexo. Resultados: Foram selecionados 137 pacientes para participarem do estudo, porém 13 foram excluídos, pois apresentavam dados incompletos, permanecendo 124 pacientes. Os fatores de risco modificáveis mais prevalentes no estudo foram sobrepeso (75,86\%) nos homens com dislipidemia e $84,48 \%$ nas mulheres com dislipidemia. 0 sedentarismo foi observado em $69 \%$ dos homens com dislipidemia e $61 \%$ das mulheres com dislipidemia. Os fatores de risco associados à dislipidemia foram a hipertensão, o diabetes mellitus e o tabagismo. Conclusões: A partir da realização deste estudo foi possível concluir que a maioria dos pacientes não tinha conhecimento prévio de seu perfil lipídico; a dislipidemia mais prevalente foi a hipertrigliceridemia isolada e o fator de risco mais prevalente foi o sobrepeso. Faz-se necessário o maior acompanhamento desses pacientes, aconselhando-os em relação a seus hábitos de vida para que possam ter uma melhor qualidade de vida.

Palavras-chave: Aterosclerose. Lipoproteínas. Lipídios. Fatores de risco.

\begin{abstract}
Introduction: Dyslipidemia is characterized by elevated plasma levels of low-density (LDL-c) cholesterol, reduced levels of high-density (HDL-c) cholesterol and / or increased triglycerides (TG). These changes evidence the risk for cardiovascular diseases (CVD) and a high probability of occurrence of death by coronary events. Objective: to evaluate the prevalence of dyslipidemia and associated risk factors in outpatients of the university hospital of the Federal University of Piauí Methods: This is a cross-sectional study, in which the sample consisted of 124 patients. The main modifiable risk factors were analyzed: overweight and sedentary lifestyle, and not modifiable: age and sex. Results: A total of 137 patients were selected to participate in the study, but 13 were excluded because they had incomplete data, remaining 124 patients. The most prevalent modifiable risk factors in the sample were overweight (75.86\%) in men with dyslipidemia and $84.48 \%$ in women with dyslipidemia. Sedentariness was observed in $69 \%$ of the men with dyslipidemia and $61 \%$ of the women with dyslipidemia. The risk factors associated with dyslipidemia were hypertension, diabetes mellitus and smoking. Conclusions: Ever since this study was carried out, it was concluded that most of the patients had no prior knowledge of their lipid profile; the most prevalent dyslipidemia was hypertriglyceridemia alone and the most prevalent risk factor was overweight. Therefore, it is necessary to follow these patients better, advising them about their lifestyle so that they can have a better quality of life.
\end{abstract}

Key words: Atherosclerosis. Lipoproteins. Lipids. Risk of factors.

\section{INTRODUÇÃO}

A dislipidemia é uma doença metabólica caracterizada pela elevação dos níveis plasmáticos de colesterol de baixa densidade (LDL-c), redução dos níveis de colesterol de alta densidade (HDL-c) e/ou aumento de triglicérides (TG). São classificadas como primárias (origem genética), ou secundárias causadas por outras doenças como: hipotireoidismo, diabetes mellitus (DM), obesidade, alcoolismo ou uso indiscriminado de medicamentos como diuréticos, betabloqueadores, corticosteroides ou anabolizantes ${ }^{1}$. As alterações no metabolismo das lipoproteínas aumentam o risco para as doenças cardiovasculares (DCV), as quais apresentam um importante desfecho crônico, sendo responsáveis por $30 \%$ do total de mortes no mundo ${ }^{2}$. Portanto, a detecção precoce das dislipidemias pode contribuir de forma efetiva para evitar o desenvolvimento prematuro de eventos coronarianos por aterosclerose ${ }^{2,3}$. Segundo o National Health and Nutrition Examination Survey (NHANES) 12,9\% da população adulta dos Estados Unidos apresentam algum tipo de dislipidemia. Um estudo envolvendo 14.385 indivíduos realizado em Shangai (China) relatou que $36,5 \%$ da população apresentava dislipidemia, com prevalências de hiperlipidemia 
mista, hipertrigliceridemia isolada, hipercolesterolemia isolada e HDL-c baixo de 3,8\%, 24,9\%, 3,2\% e 4,7\% respectivamente 3 . Já no Brasil, os estudos epidemiológicos para a investigação das dislipidemias são escassos; todavia, dados do Ministério da Saúde apontam que o Sistema Único de Saúde (SUS) gasta em média 1,3 bilhão de reais anualmente com o tratamento ambulatorial e hospitalar das dislipidemias ${ }^{4}$.

Em geral, o tratamento das dislipidemias deve ser iniciado com mudanças no estilo de vida, com hábitos alimentares saudáveis, manutenção ou aquisição de massa corporal adequada, exercícios físicos regulares, redução do tabagismo e bem-estar emocional ${ }^{4}$. Já a farmacoterapia baseia-se no uso regular de hipolipemiantes como resinas sequestrantes de ácidos biliares, fibratos, ácido nicotínico, inibidores seletivos da absorção de colesterol ou as estatinas; estes devem ser empregados em associação com o tratamento não farmacológico, sempre que o medicamento não for suficiente para alcançar as metas estabelecidas ${ }^{2,5}$.

No Brasil, as dislipidemias estão associadas a outros fatores de risco não transmissíveis, como a hipertensão arterial e a DM, que representam um grave problema de saúde pública, além de ser um dos maiores custos em assistência médica no Sistema Único de Saúde (SUS); a avaliação de 49.395 adultos residentes em capitais brasileiras e no Distrito Federal apresentou uma taxa autorreferida de dislipidemia próxima de 16,5\%, evidenciando o grande desafio a ser enfrentado pela saúde pública brasileira ${ }^{1-5}$. Diante do exposto, o objetivo desse estudo foi investigar a prevalência de dislipidemias e fatores associados em uma coorte ambulatorial.

\section{MÉTODOS}

Trata-se de um estudo quantitativo do tipo descritivo, com delineamento transversal, obtido por meio da aplicação de um questionário com pacientes ambulatoriais atendidos no laboratório de análises clínicas do hospital universitário de Teresina, PI. Foram incluídos no estudo 137 pacientes, porém 13 foram excluídos, pois apresentavam dados incompletos, permanecendo 124 pacientes de ambos os sexos, com idade entre 18 e 70 anos, os pacientes em tratamento para a dislipidemia, com incapacidade de responder aos questionamentos da entrevista ou que se recusaram a assinar o Termo de Consentimento Livre e Esclarecido. O questionário foi aplicado por duas alunas no Trabalho de Conclusão de Curso do Curso de Farmácia da Universidade Federal do Piauí. Inicialmente, na sala de espera para a coleta de sangue, foi realizada uma reunião com os participantes explicando o objetivo do trabalho. Posteriormente, após os esclarecimentos sobre o estudo, os pacientes foram convidados a responder um questionário validado contendo identificação, sexo, idade, história familiar, nível sócio econômico, escolaridade e hábitos de vida. Foram avaliados os exames de rotina realizados pelo laboratório. A coleta de dados ocorreu no período de agosto a novembro de 2015; a amostra foi selecionada por conveniência, ou seja, os sujeitos respondiam aos questionários enquanto aguardavam a coleta do sangue no laboratório de análises clínicas do hospital.

A partir da análise dos questionários e dos exames laboratoriais, os pacientes foram classificados em dislipidêmicos e não dislipidêmicos. A classificação das dislipidemias foi realizada segundo os critérios da V Diretriz Brasileira de Dislipidemias e Prevenção da Aterosclerose $(2013)^{6}$, como: Hipercolesterolemia isolada: elevação isolada do LDL-C ( $\geq 160 \mathrm{mg} / \mathrm{dL})$; Hipertrigliceridemia isolada: elevação isolada dos TG $(\geq 150$ $\mathrm{mg} / \mathrm{dL}$ ) e Hiperlipidemia mista: valores aumentados de LDL-C ( $\geq$ $160 \mathrm{mg} / \mathrm{dL}$ ), TG ( $\geq 150 \mathrm{mg} / \mathrm{dL}$ ) e HDL-C baixo: redução do HDL-C (homens $<40 \mathrm{mg} / \mathrm{dL}$ e mulheres $<50 \mathrm{mg} / \mathrm{dL}$ ) ${ }^{27}$ As variáveis pesquisadas foram: idade, sexo, escolaridade e estado civil. Em relação aos fatores de risco foram analisadas a presença ou a ausência de: Hipertensão Arterial Sistêmica, Diabetes Mellitus, sobrepeso/ obesidade, tabagismo e sedentarismo. A obesidade e o sobrepeso foram avaliados mediante o cálculo do Índice de Massa Corporal (IMC), sendo considerado como obeso o IMC $\geq 30 \mathrm{Kg} / \mathrm{m}^{2}$ e sobrepeso IMC entre 25 a $30 \mathrm{Kg} / \mathrm{m}^{2}$. O indivíduo foi classificado como sedentário quando negava prática regular de atividade física ou tinha frequência menor que duas vezes por semana. Já o tabagismo foi categorizado quando o sujeito relatava fumar um ou mais cigarros todos os dias. Em conformidade com a Resolução 466/12 do Conselho Nacional de Saúde, este estudo foi submetido ao Comitê de Ética em Pesquisa (CEP) da Universidade Federal do Piauí, obtendo-se parecer favorável à sua realização, CAAE 44530815.4.0000.5214. Os dados foram analisados e as figuras plotadas utilizando-se o software Microsoft Office Excel $2007^{\circledR}$. A análise estatística foi realizada no programa SPSS, versão 20.

\section{RESULTADOS}

A média de idade dos participantes da pesquisa foi de $57,35 \pm 1,85$ entre os homens e 50,32 $\pm 17,2$ entre as mulheres. Em relação ao grau de instrução, a maioria dos indivíduos declarou possuir o ensino fundamental completo (Tabela 1). Verificou-se que $58,62 \%$ dos homens não sabiam ter a doença, e $34,48 \%$ das mulheres relataram não saber da existência da dislipidemia (Figura 1).

Na Figura 2, é possível verificar o perfil de distribuição dessa classificação entre homens e mulheres. Observou-se que 91,38\% das mulheres e $75,86 \%$ dos homens tinham HDL baixo. Quanto aos níveis séricos de triglicerídeos $44,82 \%$ dos homens e $39,65 \%$ das mulheres apresentaram níveis elevados.

Entre os indivíduos com dislipidemia que não apresentavam sobrepeso/obesidade, foi verificada a presença de pelo menos um fator de risco associado à dislipidemia em que 43,1\% tinham hipertensão e 15,51\% DM. Na amostra em estudo, o percentual de homens com dislipidemia sem nenhum fator de risco foi de 97,1\%; já entre as mulheres com dislipidemia, o percentual que não apresentava nenhum fator de risco foi de $87,6 \%(p=0,007)$. Todavia, não possível observar uma associação significativa entre os fatores de risco e a prevalência de dislipidemia (Tabela 2). 
Tabela 1. Perfil sociodemográfico dos participantes do estudo, segundo o gênero

\begin{tabular}{|c|c|c|c|c|}
\hline \multirow[t]{2}{*}{ Características } & \multicolumn{2}{|c|}{$\begin{array}{r}\text { Homens } \\
(n=42)\end{array}$} & \multicolumn{2}{|c|}{$\begin{array}{r}\text { Mulheres } \\
\quad(n=82)\end{array}$} \\
\hline & $\mathbf{N}$ & $\%$ & $\mathbf{N}$ & $\%$ \\
\hline \multicolumn{5}{|l|}{ Características gerais } \\
\hline Média de idade & $57,35 \pm 18,5$ & & $50,32 \pm 17,2$ & \\
\hline Analfabetismo & 5 & 11,9 & 8 & 9,8 \\
\hline Ensino Fundamental & 24 & 57,1 & 33 & 40,2 \\
\hline Ensino Médio & 12 & 28,6 & 32 & 39 \\
\hline Ensino Superior & 1 & 2,4 & 9 & 11 \\
\hline $\begin{array}{l}\text { Casado ou em união } \\
\text { estável }\end{array}$ & 32 & 76,2 & 35 & 42,7 \\
\hline $\begin{array}{l}\text { Solteiro, divorciado ou } \\
\text { viúvo }\end{array}$ & 10 & 23,8 & 47 & 57,3 \\
\hline
\end{tabular}

Figura 1. Prevalência de indivíduos que relataram ter dislipidemia.

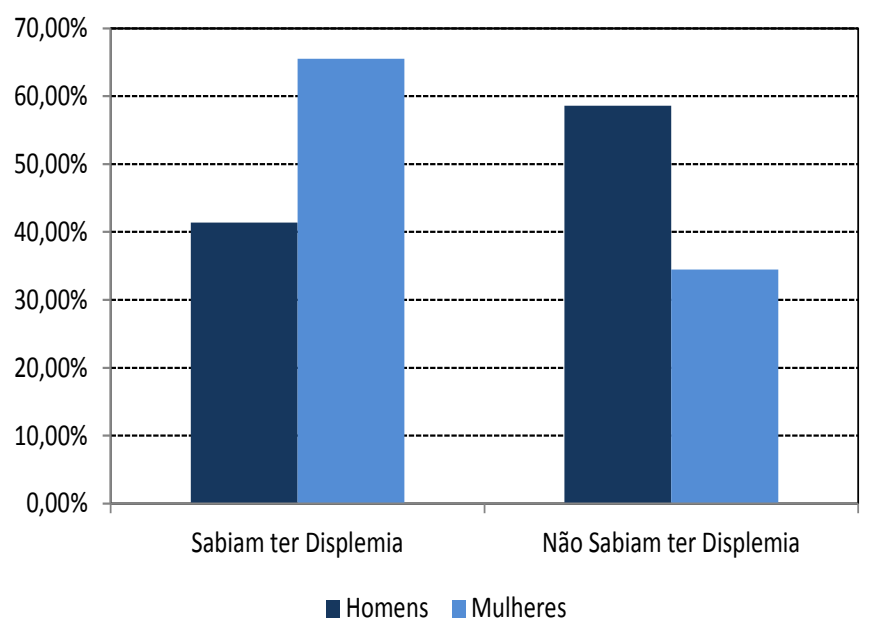

Figura 2. Classificação laboratorial das dislipidemias, segundo o gênero.

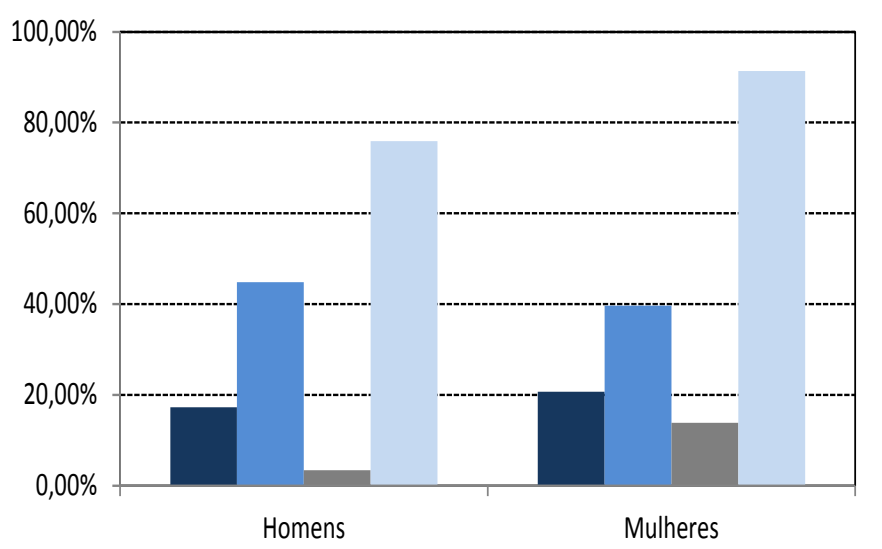

- Hipercolesterolemia isolada -Hipertrigliceridemia isolada - Hiperlipidemia mista $\quad$ HDLC-baixo

\section{DISCUSSÃO}

No Brasil, as Doenças Crônicas Não Transmissíveis (DCNT) representam um grande problema de saúde pública. Para que seja possível monitorar os comportamentos de risco que levam a população a desenvolver tais doenças, é preciso que se tenha o conhecimento sobre o perfil de exposição populacional a seus fatores de risco. Os quatro principais grupos de DCNT são: as doenças circulatórias, e respiratórias crônicas, o câncer e a diabetes. Seus principais determinantes modificáveis, ou fatores de risco (FR) em comum são: tabagismo, álcool, inatividade física, alimentação não saudável e obesidade? ${ }^{7}$.

Entende-se como fator de risco as situações ou doenças que aumentam o risco de desenvolvimento de determinada doença. A probabilidade de ocorrência de eventos relacionados à desordem no metabolismo lipídico aumenta com a presença de múltiplos fatores de risco. Para a dislipidemia, sexo e idade são fatores de risco não modificáveis significativos, pois os níveis de lipídios e lipoproteínas sofrem variações importantes durante a fase de crescimento humano, com diferenças segundo idade e sexo. Em adolescentes do sexo feminino o LDL-c e o CT elevamse progressivamente a partir dos 14-15 anos, sendo superiores aos dos meninos por volta dos 17-18 anos 8 .

Segundo a Pesquisa Nacional de Saúde (PNS) 2014, 78\% das mulheres foram ao médico nos últimos 12 meses; já em relação aos homens, essa porcentagem caiu para 63,9\%, achado semelhante aos nossos resultados em que o percentual de mulheres que sabiam ter dislipidemia foi de $65,52 \%$ e dos homens 41,38\% (Figura 1), sabe-se, contudo, que o desconhecimento da doença impossibilita seu tratamento e possível controle ${ }^{11}$.

A Figura 2 retrata as dislipidemias entre homens e mulheres; em ambos os gêneros houve prevalência da hipertrigliceridemia isolada; leucócitos de pacientes com hipertrigliceridemia liberam maiores quantidades de espécies reativas de oxigênio, quando comparados aos indivíduos normolipidêmicos. O aumento da produção dessas espécies reativas de oxigênio pelos leucócitos, nos portadores de hiperlipidemia combinada e sua relação positiva com os níveis de triglicerídeos, pode contribuir para o esclarecimento dos mecanismos envolvidos na aterogenicidade das lipoproteínas ricas em triglicerídeos. Assim, entende-se que a hipertrigliceridemia atua sinergicamente aumentando o risco de desenvolvimento da DAC associada à hipercolesterolemia ${ }^{3,12}$. $\mathrm{Na}$ amostra estudada, a dislipidemia mista foi a de menor prevalência: 3,41\% nos homens e 13,79\% nas mulheres (Figura 2).

Altas concentrações sanguíneas de LDL-c permitem sua entrada no endotélio vascular e, em seguida, penetram na parede da artéria. A LDL-c sofre oxidação na camada da íntima e como consequência ocorre a formação de placas ateroscleróticas culminando na doença cardiovascular. A lipoproteína de baixa densidade ( $L D L$ ) é reconhecidamente envolvida na aterogênese, tanto por suas alterações quantitativas, como qualitativas. Os 
Tabela 2. Prevalência de dislipidemia associada a fatores de risco

\begin{tabular}{|c|c|c|c|c|c|c|}
\hline \multirow{3}{*}{ Fator de Risco } & \multirow{3}{*}{ Total } & \multicolumn{4}{|c|}{ Prevalência de dislipidemia } & \multirow{3}{*}{ Valor de $p$} \\
\hline & & \multirow[b]{2}{*}{$\mathbf{n}$} & Masculino & \multicolumn{2}{|c|}{ Feminino } & \\
\hline & & & $\%$ & $\mathbf{n}$ & $\%$ & \\
\hline Sedentarismo & & & & & & 0,658 \\
\hline Sim & 79 & 29 & 28,2 & 50 & 25,8 & \\
\hline Não & 218 & 74 & 71,8 & 144 & 74,2 & \\
\hline Odds Ratio (IC 95\%) & & 1 & $1,13(0,66-1,93)$ & & & \\
\hline Sobrepeso & & & & & & 0,436 \\
\hline Sim & 101 & 32 & 31,1 & 69 & 35,6 & \\
\hline Não & 196 & 71 & 68,9 & 125 & 64,4 & \\
\hline Odds Ratio (IC 95\%) & & 1 & $0,82(0,49-1,36)$ & & & \\
\hline Hipertensão & & & & & & 0,203 \\
\hline Sim & 60 & 25 & 24,3 & 35 & 18,0 & \\
\hline Não & 237 & 78 & 75,7 & 159 & 82,0 & \\
\hline Odds Ratio (IC 95\%) & & 1 & $1,46(0,82-2,60)$ & & & \\
\hline Diabetes & & & & & & 0,191 \\
\hline Sim & 22 & 10 & 9,7 & 12 & 6,2 & \\
\hline Não & 275 & 93 & 90,3 & 182 & 93,8 & \\
\hline Odds Ratio (IC 95\%) & & 1 & $1,63(0,68-3,92)$ & & & \\
\hline Tabagismo & & & & & & 0,352 \\
\hline Sim & 8 & 4 & 3,9 & 4 & 2,1 & \\
\hline Não & 289 & 99 & 96,1 & 190 & 97,9 & \\
\hline Odds Ratio (IC 95\%) & & 1 & $1,92(0,47-7,84)$ & & & \\
\hline Sem fator de risco & & & & & & 0,007 \\
\hline Sim & 27 & 3 & 2,9 & 24 & 12,4 & \\
\hline Não & 270 & 100 & 97,1 & 170 & 87,6 & \\
\hline Odds Ratio (IC 95\%) & & 1 & $0,21(0,06-0,72)$ & & & \\
\hline
\end{tabular}

níveis de LDL-c iguais ou superiores a $160 \mathrm{mg} / \mathrm{dL}$ estão associados ao maior risco de desenvolvimento de doença cardiovascular. A propósito, essa lipoproteína é a partícula lipoprotéica mais aterogênica, sendo mais susceptível a modificações, como a oxidação e sua posterior captação pelos macrófagos e a consequente transformação destes em células espumosas ${ }^{13}$. No presente estudo, o percentual de hipercolesterolemia isolada em mulheres foi de $20,65 \%$, sendo maior que o percentual em homens 17,24\% (Figura 2).

Partículas de HDL podem facilitar o transporte reverso de colesterol, em que frações de HDL adquirem o colesterol dos tecidos periféricos, transportando-os de volta para o fígado. Daí sua importância no ateroma, a fim de mediar este mesmo processo em placas ateroscleróticas, removendo o colesterol, gordura da parede da artéria e retornando-a para o fígado. Todavia, nas dislipidemias, o baixo nível de HDL acompanhado pela elevação dos níveis de LDL-c predispõem essas lipoproteínas a um tempo maior na corrente sanguínea, resultando frequentemente na oxidação dessas moléculas14.
O nível de HDLc-baixo na população estudada foi significativo, chegando a $75,86 \%$ nos homens e 91,385 nas mulheres (Figura 2).

O sobrepeso e/ou a obesidade são considerados fatores de risco de primeira linha para várias comorbidades, pois estão associados a outros fatores de risco como hipertensão (HAS), dislipidemia e resistência à insulina, além de serem fatores de risco que mais aumentam em prevalência. Assim como no sobrepeso, a parcela de indivíduos dislipidêmicos sedentários foi superior à dos indivíduos dislipidêmicos que praticavam algum tipo de atividade física. $69 \%$ dos homens que tinham dislipidemia eram sedentários; entre as mulheres, $61 \%$ não faziam nenhuma atividade física. $A$ atividade física de intensidade moderada a alta possui importante impacto sobre o perfil lipídico. Quanto maior a intensidade e a regularidade dessa atividade, melhor o perfil lipídico ${ }^{15}$. A prática regular de exercícios físicos proporciona alterações anti-aterogênicas significativas nos níveis séricos dos lipídios, ocorrendo principalmente aumento de HDL e redução da LDL-c e, consequentemente de triglicerídeos, além 
de aumentar a tolerância à glicose, a sensibilidade à insulina e diminuir a agregação plaquetária ${ }^{16}$.

Apesar do crescente conhecimento a respeito da atividade física, a inatividade física tem sido um dos fatores de risco mais frequentes na maioria dos estudos. Comportamentos adaptativos, como a atividade física moderada reduz risco de desenvolver dislipidemia. Em estudos preliminares, a redução da atividade física tem sido a mais importante contribuinte para a obesidade ${ }^{17}$.

Neste estudo, a HAS foi o fator de risco mais prevalente nos pacientes com dislipidemia, DM e tabagismo, respectivamente. Em ambos os sexos, o percentual de indivíduos dislipidêmicos com hipertensão foi o mais elevado; entre esses fatores de risco encontrados, $58,62 \%$ foram em homens e $43,10 \%$ em mulheres. A HAS é uma das doenças crônicas mais prevalentes em adultos, fato que a tornou uma prioridade e um grande desafio em saúde pública. Além disso, é o segundo principal fator de risco para as doenças cardiovasculares em adultos e o primeiro fator de risco para os acidentes vasculares cerebrais. Vale ressaltar, também, que o primeiro fator de risco para o desenvolvimento de doenças cardiovasculares são as dislipidemias ${ }^{19,26}$. Os níveis pressóricos são mais elevados em pacientes com excesso de peso. Atualmente, a prevalência de HAS em indivíduos com excesso de peso é 2,9 vezes maior quando comparada a indivíduos eutróficos. Estudos têm revelado que o fator mais importante na gênese da hipertensão na infância e na adolescência é a obesidade ${ }^{13,20}$.

ODM, outrofator de risco de importância para o desenvolvimento das dislipidemias, teve um percentual de $24,14 \%$ nos homens com dislipidemia, e 15,51\% nas mulheres dislipidêmicas. Esta doença vem tomando proporções epidêmicas em razão do aumento da expectativa de vida, do crescimento da prevalência de obesidade e dos hábitos de vida sedentária. Estudos vêm destacando que a resistência à insulina é o principal fator na patogênese do Diabetes tipo 2, além de ser um cofator no desenvolvimento da HAS, dislipidemia e aterosclerose ${ }^{21,22,26}$.

O tabagismo foi o fator de risco menos predominante em nossos achados, tanto para os homens $(10,34 \%)$ quanto para as mulheres (5,17\%). O fumo aumenta os níveis de LDL-c, de VLDL e triglicérides, além de reduzir os níveis de HDL, pois aumenta a adesividade plaquetária, lesiona o endotélio arterial e contribui para a elevação da pressão sanguínea24,25,26. Dessa forma, os fumantes tendem a apresentar prevalência 2,5 vezes maior de hipercolesterolemia quando comparados aos não fumantes ${ }^{23}$.

\section{CONCLUSÃO}

Houve uma alta prevalência de dislipidemia na demanda ambulatorial do hospital universitário. A dislipidemia foi mais prevalente entre as mulheres e em faixa etárias mais avançadas. Pôde-se concluir ainda que uma grande parcela da população em estudo não tinha conhecimento prévio de seu perfil lipídico, que a dislipidemia mais prevalente foi a hipertrigliceridemia isolada e o fator de risco mais prevalente foi o sobrepeso. Portanto, faz-se necessário o maior acompanhamento desses pacientes, aconselhando-os em relação a seus hábitos de vida para que possam ter uma melhor qualidade de vida.

\section{REFERÊNCIAS}

1. Sociedade Brasileira de Cardiologia, Sociedade Brasileira de Hipertensão, Sociedade Brasileira de Nefrologia. VI Diretrizes Brasileiras de Hipertensão Arterial. Arquivos Brasileiros de Cardiologia. 2010; 95 (1): 1-3. doi: http://dx.doi. org/10.1590/S0066-782X2010001700001.

2. Rodrigues FM, Petruzellis EA, Bock PM. Avaliação do perfil de usuários de hipolipemiantes de uma drogaria em Porto Alegre RS. Rev Saúde (Santa Maria). 2013; 39(2): 149-160. doi: http://dx.doi.org/10.5902/223658347255.

3. Moraes AS, Checchio MV, Freitas, ICM. Dislipidemia e fatores associados em adultos residentes em Ribeirão Preto, SP: resultados do Projeto EPIDCV. Arq Bras Endocrinol Metab.2013 Dez; 57(9): 691-701. doi: http://dx.doi.org/10.1590/ S0004-27302013000900004.

4. Miname MH, Mangili L, Santos RD. Tratamento das dislipidemias. Rev Clínica médica: diagnóstico e tratamento. 2013; (3): 2407-2427.

5. Abadi BL, Budel JM. Aspectos Clínicos Laboratoriais das Dislipidemias. Cadernos da Escola de Saúde. 2011; 5: 158-169.

6. Sociedade Brasileira de Cardiologia. V Diretriz Brasileira de Dislipidemias e Prevenção da Aterosclerose. Arq Bras Cardiol. 2013; 101(4):1-36.

7. Grupo de Investigación en Atención Farmacéutica, Universidad de Granada Grupo de Investigacion Pharmaceutica Care España, Sociedad Española de Farmacia Comunitaria. Tercer consenso de granada sobre problemas relacionados con medicamentos (PRM) y resultados negativos asociados a lamedicación (RNM). Ars Pharm. 2007; 48(1): 5-7.
8. Carvalho FD. Avaliação econômica do impacto da atividade de atenção farmacêutica na assistência à saúde: aspectos metodológicos [tese]. São Paulo (SP): Universidade de São Paulo; 2012.

9. Ministério da Saúde (BR). Plano de ações estratégicas para enfrentamento das doenças crônicas não transmissíveis (DCNT) no Brasil 2011-2022. Brasília: Ministério da Saúde; 2011.

10. Morrison JA, Sprecher DL, Biro FM, Apperson-hansen C, Dipaola LM. Serum testosterone associates with lower high-density lipoprotein cholesterol in black and white males, 10 to 15 years of age, through lowered apolipoprotein Al and All concentrations. Metabolism. 2002 Apr; 51(4): 432-437. PubMed PMID: 11912549.

11. Szwarcwald CL, Malta DC, Pereira CA, Vieira MLFP, Conde WL, Souza PRB Jr, et al. Pesquisa Nacional de Saúde no Brasil: concepção e metodologia de aplicação. Ciênc. Saúde coletiva. 2014 Fev; 19(2):333-342. doi: http://dx.doi. org/10.1590/1413-81232014192.14072012.

12. Salonen JT, Yla-Herttuala S, Yamamoto R, Butler S, Korpela, H.; Salonem R, et al. Autoanibody against oxidized LDL and progression of carotid atherosclerosis. Lancet.1992 Apr. 339(8798): 883-891. PubMed PMID: 1348295.

13. Barbosa MDCL, Barbosa, JB, Guerra, LFA, Barbosa, MFL, Barbosa FL, Barbosa RL, et al. Dislipidemia e risco cardiovascular em afrodescendentes: um estudo em comunidades quilombolas do Maranhão, Brasil. Rev Bras Med Fam Comunidade. 2015 Jul-Set; 10 (36). doi: http://dx.doi.org/10.5712/ rbmfc10(36)925. 
14. Prassl R, Laggner P. Lipoprotein structure and dynamics: low density lipoprotein viewed as a highly dynamic and flexible nanoparticle. In Frank $S$, Kostner G, editors. Lipoproteins: role in health and diseases. Rijeka: Intech; 2012. Cap.1.

15. Li J, Siegrist J. Physical Activity and risk of cardiovascular disease -- a metaanalysis of prospective cohort studies. Int J Environ Res Public Health. 2012 Feb; 9(2):391-407. doi: 10.3390/ijerph9020391.

16. Accioly MF, Padulla SAT, Carmo EM, Camargo JCS Filho, Brandão AC, Souza DRS. Efeito do treinamento físico aeróbio e do uso de estatinas sobre o perfil lipídico de animais com dislipidemia. R. bras Ci e Mov. 2016; 24(1): 108-117.

17. Teo K, Chow CK, Vaz M, Rangarajan S, Yusuf S, PURE Investigators-Writing Group. The Prospective Urban Rural Epidemiology (PURE) study: examining the impact of societal influences on chronic non communicable diseases in low , middle-, and high-income countries. Am Heart J. 2009 Jul; 158(1): 1-7. doi: 10.1016/j.ahj.2009.04.019.

18. Pinho L, Aguiar APS, Oliveira MR, Barreto NAP, Ferreira CMM. Hipertensão e dislipidemia em pacientes diabetes mellitus tipo 2: uma revisão integrativa. Renome.2015; 4(1): 87-101.

19. Reuter CP, Silva PTD, Renner JDP, Mello EDD, Valim ARDM, Pasa L, et al. Dislipidemia associa-se com falta de aptidão e sobrepeso-obesidade em crianças e adolescentes. Arq Bras Cardiol. 2016;106(3). doi: 10.5935/abc.20160025.

20. Franca E, Alves JG. Dislipidemia entre crianças e adolescentes de
Pernambuco. Arq Bras Cardiol. 2006; 87(6): 722-727.

21. Dessi M, Noce A, Bertucci P, Di Villahermosa SM, Zenobi R, Castagnola V, et al. Atherosclerosis, dyslipidemia, and inflammation: the significant rola of polyunsaturated fatty acids. ISRN Inflamm. 2013 May; 2013: 191823.

22. Hanson K, Allen S, Jensen S, Hatsukami D. Treatment of adolescent smokers with the nicotine patch. Nicotine Tob Res. 2003 Aug; 5(4): 515-526. doi: 10.1080/1462220031000118559. PubMed PMID: 12959789.

23. Gus I, Ribeiro RA, Kato S, Basos J, Medina C, Zazlavsky C, et al. Variações na prevalência dos fatores de risco para doença arterial coronariana no Rio Grande do Sul: uma análise comparativa entre 2002-2014. Arq Bras Cardiol. 2015; 105(6): 573-579. doi: 10.5935/abc.20150127.

24. Lee JM, Choudhury RP. Atherosclerosis regression and high-density lipoproteins. Expert RevCardiovascTher.2010 Sep; 8(9): 1325-1334. doi: 10.1586/erc.10.108.

25. LESSA I. Doenças crônicas não-transmissíveis no Brasil: um desafio para a complexa tarefa de vigilância. Ciênc. saúde colet 2004; 9(4): 931-943. doi: http://dx.doi.org/10.1590/S1413-81232004000400014.

26. Xavier HT, Izar MC, Faria Neto JR, Assad MH, Rocha VZ, Sposito AC, et al, coordenadores. $V$ Diretriz brasileira de dislipidemias e prevenção da aterosclerose. . Arq Bras Cardiol. 2013; 101(4): 1-20. 